\title{
A note on 2-D lithospheric deformation due to a blind strike-slip fault
}

\author{
Sunita Rani and SaRva Jit Singh ${ }^{2}$ \\ ${ }^{1}$ Department of Applied Mathematics, Guru Jambheshwar University, Hisar 125 001, India. \\ e-mail: s_b_rani@rediffmail.com \\ ${ }^{2}$ Department of Mathematics, University of Delhi, South Campus, New Delhi 110 021, India. \\ e-mail: s_j_singh@yahoo.com
}

\begin{abstract}
Analytical solution for the problem of a surface-breaking long strike-slip fault in an elastic layer overlying an elastic half-space is well known. The purpose of this note is to obtain the corresponding solution for a blind fault. Since the solution is valid for arbitrary values of the fault-depth and the dip angle, the effects of these two important fault parameters can be studied numerically. The variation of the parallel displacement and shear stress with the distance from the fault is studied numerically for different values of the fault-depth and dip angle.
\end{abstract}

\section{Introduction}

A complete understanding of the processes involved in an earthquake cycle requires knowledge of the distribution of displacements in a region and how they vary with time in the epochs preceding an earthquake, during an earthquake and following an earthquake. We may recast these epochs in terms of strain accumulation prior to an earthquake, coseismic deformation and postseismic deformation. Several researchers have developed models of coseismic lithospheric deformation. Rybicki (1971) found a closed-form analytical solution for the problem of a long vertical strike-slip fault in a two-layer model of the earth. Chinnery and Jovanovich (1972) extended the solution to a three-layer model. Savage and Prescott (1978) constructed a simple two-dimensional model of an earthquake cycle that takes place on a transform fault.

Singh and Rani (1994) obtained an analytical solution for the problem of a long inclined strikeslip fault in an elastic layer overlying an elastic half-space. In the model used by Singh and Rani (1994), the depth $d$ of the upper edge of the fault does not occur explicitly in the solution. Moreover, as the dip angle tends to zero, the fault approaches the surface of the earth. Therefore, their results cannot be used to study the deformation of a twolayer model caused by deep strike-slip faults of small dip angles. The purpose of the present note is to obtain a closed-form analytical solution for the problem of a long blind strike-slip fault located in an elastic layer overlying an elastic half-space. The depth $d$ occurs explicitly in the solution. Therefore, the effect of the variations in the depth for a fixed dip and vice versa can be studied directly. Figures showing the effect of variations in the depth $d$ on the surface displacement and shear stress are presented.

\section{Theory}

We consider an earth model consisting of a homogeneous, isotropic, elastic layer of thickness $H$ overlying a homogeneous, isotropic, elastic half-space (figure 1). We place the origin of a Cartesian coordinate system $\left(x_{1}, x_{2}, x_{3}\right)$ at the free boundary and the $x_{3}$-axis points downwards into the medium. A long inclined strike-slip fault, with strike along the $x_{1}$-axis, is situated in the layer. The upper edge of

Keywords. Blind fault; coseismic deformation; strike-slip fault; two-dimensional. 
the fault is taken to be at depth $d$. Let $\mu_{1}$ and $\mu_{2}$ be the rigidities of the layer and of the half-space, respectively. The superscript (1) denotes quantities related to the layer and the superscript (2) denotes quantities related to the half-space.

Following Singh and Rani (1994), we obtain the following expressions for the displacements in the strike direction ( $x_{1}$-direction) due to an inclined strike-slip fault of finite width $L$ and infinite length under the assumption of antiplane strain problem: where $\delta$ is the dip angle, $s$ is the distance from the upper edge of the fault measured in the down-dip direction (figure 1) and

$$
\begin{aligned}
x & =x_{3}-d, \quad x^{\prime}=x_{3}+d, \\
\left.f(s)\right|_{0} ^{L} & =f(L)-f(0),
\end{aligned}
$$

$$
\begin{aligned}
u^{(1)}= & \frac{1}{2 \pi}\left[M_{0}\left\{\tan ^{-1}\left(\frac{s-x_{2} \cos \delta-x \sin \delta}{x \cos \delta-x_{2} \sin \delta}\right)-\tan ^{-1}\left(\frac{s-x_{2} \cos \delta+x^{\prime} \sin \delta}{x^{\prime} \cos \delta+x_{2} \sin \delta}\right)\right\}\right. \\
& +\sum_{n=1}^{\infty} M_{n}\left\{\tan ^{-1}\left(\frac{s-x_{2} \cos \delta+x^{\prime} \sin \delta-2 n H \sin \delta}{2 n H \cos \delta-x^{\prime} \cos \delta-x_{2} \sin \delta}\right)+\tan ^{-1}\left(\frac{s-x_{2} \cos \delta-x \sin \delta-2 n H \sin \delta}{2 n H \cos \delta+x \cos \delta-x_{2} \sin \delta}\right)\right. \\
& \left.\left.-\tan ^{-1}\left(\frac{s-x_{2} \cos \delta-x \sin \delta+2 n H \sin \delta}{2 n H \cos \delta-x \cos \delta+x_{2} \sin \delta}\right)-\tan ^{-1}\left(\frac{s-x_{2} \cos \delta+x^{\prime} \sin \delta+2 n H \sin \delta}{2 n H \cos \delta+x^{\prime} \cos \delta+x_{2} \sin \delta}\right)\right\}\right]\left.\right|_{0} ^{L} \\
u^{(2)}= & \frac{1}{\pi}\left[N_{0}\left\{\tan ^{-1}\left(\frac{s-x_{2} \cos \delta-x \sin \delta}{x \cos \delta-x_{2} \sin \delta}\right)-\tan ^{-1}\left(\frac{s-x_{2} \cos \delta+x^{\prime} \sin \delta}{x^{\prime} \cos \delta+x_{2} \sin \delta}\right)\right\}\right. \\
& \left.+\sum_{n=1}^{\infty} N_{n}\left\{\tan ^{-1}\left(\frac{s-x_{2} \cos \delta-x \sin \delta-2 n H \sin \delta}{2 n H \cos \delta+x \cos \delta-x_{2} \sin \delta}\right)-\tan ^{-1}\left(\frac{s-x_{2} \cos \delta+x^{\prime} \sin \delta+2 n H \sin \delta}{2 n H \cos \delta+x^{\prime} \cos \delta+x_{2} \sin \delta}\right)\right\}\right]\left.\right|_{0} ^{L},
\end{aligned}
$$

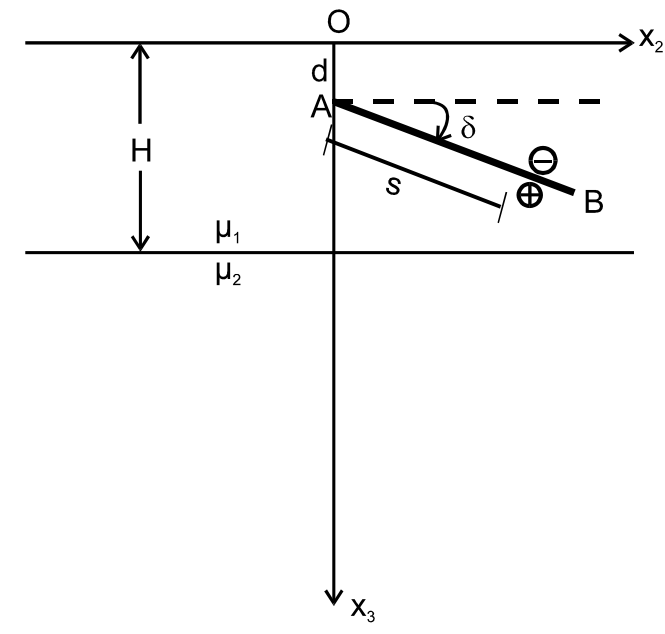

Figure 1. Geometry of a long strike-slip fault of width $L$ situated in a layer of uniform thickness $H$ overlying a half-space. The displacement discontinuity (slip) on the fault is parallel to the $x_{1}$-axis. The sign $\oplus$ indicates displacement in the direction of the $x_{1}$-axis, the $\operatorname{sign} \ominus$ in the opposite direction. $d$ is the depth of the upper edge $A$ of the fault and $\delta$ is the dip angle. $s$ is the distance from the upper edge of the fault measured in the down-dip direction. $\mu_{1}$ and $\mu_{2}$ denote the rigidity of the layer and the half-space, respectively.

$$
\begin{aligned}
& M_{n}=b\left(\frac{\mu_{1}-\mu_{2}}{\mu_{1}+\mu_{2}}\right)^{n}, \\
& N_{n}=b \mu_{1} \frac{\left(\mu_{1}-\mu_{2}\right)^{n}}{\left(\mu_{1}+\mu_{2}\right)^{n+1}},
\end{aligned}
$$

$d$ being the depth of the upper edge of the fault and $b$ the slip. The nonzero stresses are given by

$$
p_{12}^{(i)}=\mu_{i} \frac{\partial u^{(i)}}{\partial x_{2}}, \quad p_{13}^{(i)}=\mu_{i} \frac{\partial u^{(i)}}{\partial x_{3}}
$$

(no summation over $i ; i=1,2$ ).

From equations (1), (2) and (7), we get the following expressions for the stresses 

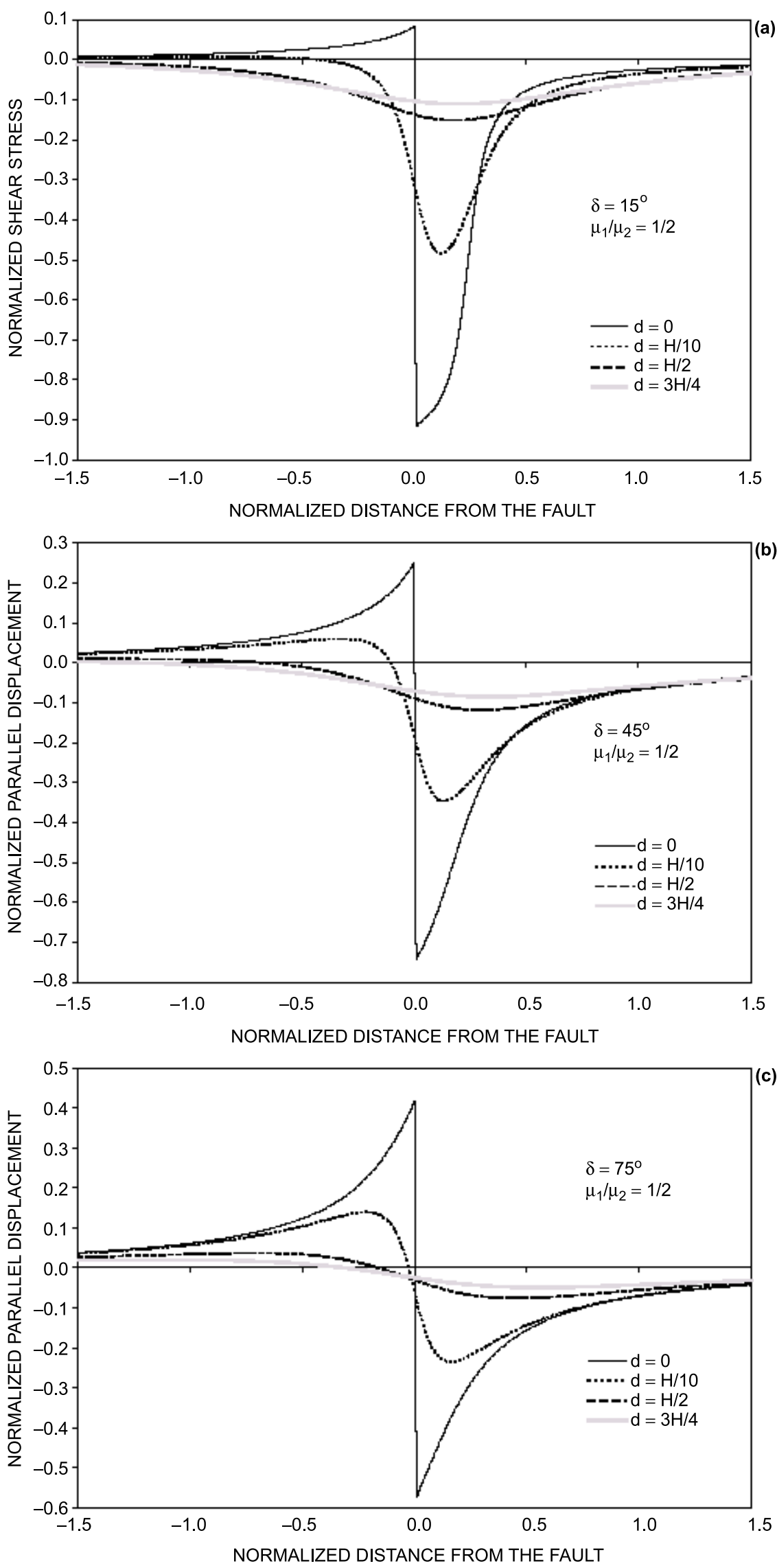

Figure 2. Variation of the normalized parallel displacement $(u / b)$ at the surface with the normalized distance from the fault $\left(x_{2} / H\right)$ assuming $\mu_{1} / \mu_{2}=1 / 2$ for four different values of depth $d=0, H / 10, H / 2$ and $3 H / 4$ for (a) $\delta=15^{\circ}$; (b) $\delta=45^{\circ}$; (c) $\delta=75^{\circ}$. 

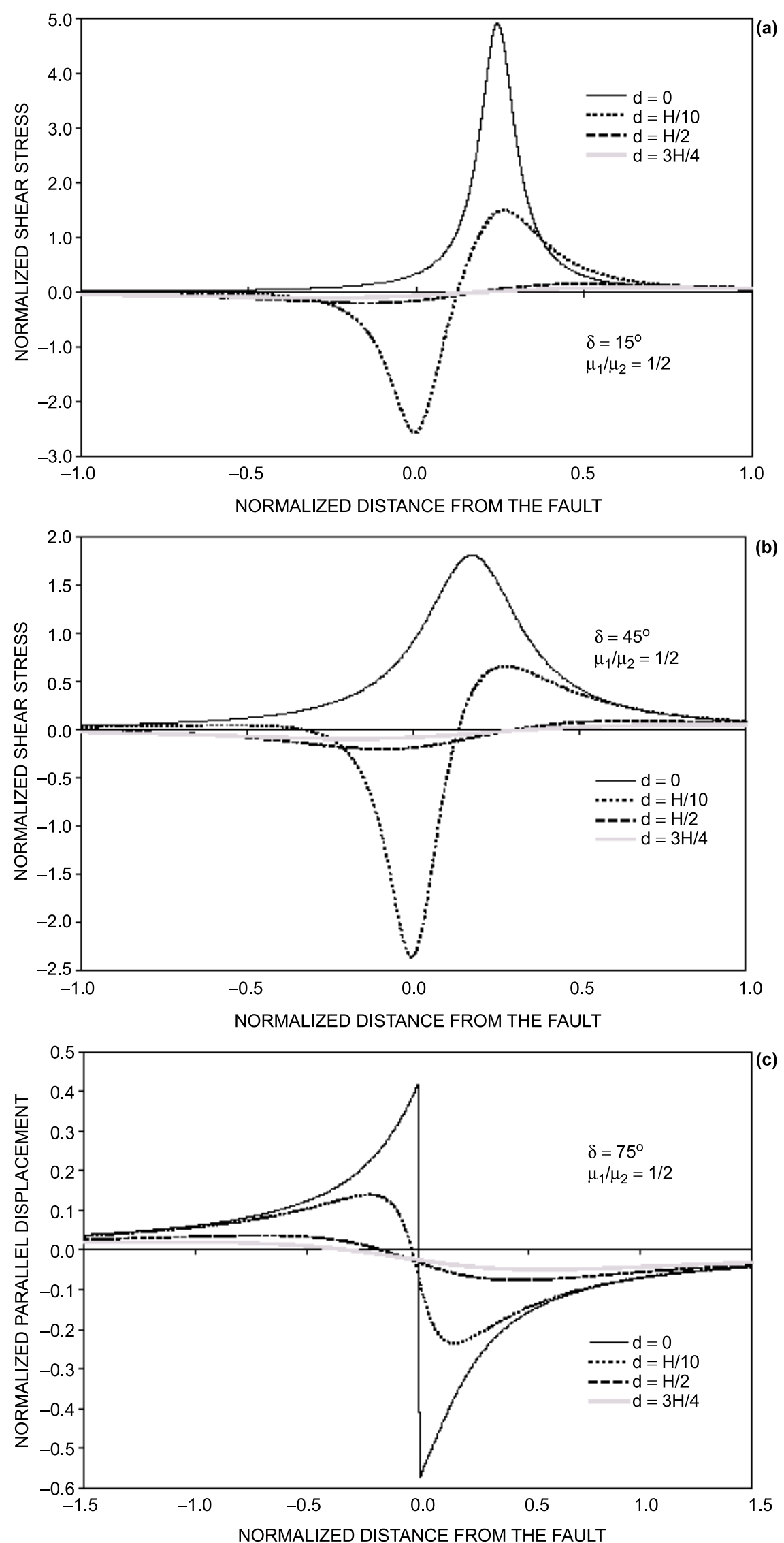

Figure 3. Variation of the normalized shear stress $P_{12}\left[=p_{12} /\left(\mu_{1} b / H\right)\right]$ at the surface with the normalized distance from the fault $\left(x_{2} / H\right)$ assuming $\mu_{1} / \mu_{2}=1 / 2$ for four different values of $d=0, H / 10, H / 2$ and $3 H / 4$ for (a) $\delta=15^{\circ}$; (b) $\delta=45^{\circ}$; (c) $\delta=75^{\circ}$. 


$$
\begin{aligned}
p_{12}{ }^{(1)}= & \frac{\mu_{1}}{2 \pi}\left[M_{0}\left(-\frac{x-s \sin \delta}{R^{2}}+\frac{x^{\prime}+s \sin \delta}{S^{2}}\right)\right. \\
& +\sum_{n=1}^{\infty} M_{n}\left(\frac{x^{\prime}+s \sin \delta-2 n H}{T^{2}}\right. \\
& -\frac{x-s \sin \delta+2 n H}{V^{2}}-\frac{x-s \sin \delta-2 n H}{U^{2}} \\
& \left.\left.\left.+\frac{x^{\prime}+s \sin \delta+2 n H}{W^{2}}\right)\right]|,| \frac{1}{0}\right) \\
p_{13}(1)= & \mu_{1} \frac{\left(x_{2}-s \cos \delta\right)}{2 \pi}\left[M_{0}\left(\frac{1}{R^{2}}-\frac{1}{S^{2}}\right)\right. \\
& \left.+\sum_{n=1}^{\infty} M_{n}\left(-\frac{1}{T^{2}}+\frac{1}{V^{2}}+\frac{1}{U^{2}}-\frac{1}{W^{2}}\right)\right]
\end{aligned}
$$

$$
\begin{aligned}
p_{12}{ }^{(2)}= & \frac{1}{\pi}\left[P_{0}\left(-\frac{x-s \sin \delta}{R^{2}}+\frac{x^{\prime}+s \sin \delta}{S^{2}}\right)\right. \\
& +\sum_{n=1}^{\infty} P_{n}\left(-\frac{x-s \sin \delta+2 n H}{V^{2}}\right. \\
& \left.\left.+\frac{x^{\prime}+s \sin \delta+2 n H}{W^{2}}\right)\right]\left.\right|_{0} ^{L},
\end{aligned}
$$$$
p_{13}^{(2)}=\frac{\left(x_{2}-s \cos \delta\right)}{\pi}\left[P_{0}\left(\frac{1}{R^{2}}-\frac{1}{S^{2}}\right)\right.
$$$$
\left.+\sum_{n=1}^{\infty} P_{n}\left(\frac{1}{V^{2}}-\frac{1}{W^{2}}\right)\right]\left.\right|_{0} ^{L}
$$

where

$$
\begin{aligned}
& R^{2}=\left(x_{2}-s \cos \delta\right)^{2}+(x-s \sin \delta)^{2} \\
& S^{2}=\left(x_{2}-s \cos \delta\right)^{2}+\left(x^{\prime}+s \sin \delta\right)^{2}, \\
& T^{2}=\left(x_{2}-s \cos \delta\right)^{2}+\left(2 n H-x^{\prime}-s \sin \delta\right)^{2}, \\
& U^{2}=\left(x_{2}-s \cos \delta\right)^{2}+(2 n H-x+s \sin \delta)^{2}, \\
& V^{2}=\left(x_{2}-s \cos \delta\right)^{2}+(2 n H+x-s \sin \delta)^{2},
\end{aligned}
$$

$$
\begin{aligned}
W^{2} & =\left(x_{2}-s \cos \delta\right)^{2}+\left(2 n H+x^{\prime}+s \sin \delta\right)^{2} \\
P_{n} & =b \mu_{1} \mu_{2} \frac{\left(\mu_{1}-\mu_{2}\right)^{n}}{\left(\mu_{1}+\mu_{2}\right)^{n+1}} .
\end{aligned}
$$

On substituting $d=0$ in equations (1), (2), and (8) to (11) we obtain the corresponding results for a surface-breaking fault given by Singh and Rani (1994).

\section{Numerical results}

To examine the effect of the variation in the depth $d$ of the upper edge of the fault on the coseismic field, we assume $\mu_{1} / \mu_{2}=1 / 2$ and $L=H / 4$. The explicit analytical expressions giving the elastic field involve infinite series. The infinite series appearing in the right-hand side of equations (1), (2), and (8) to (11) converge very rapidly. Numerically, it is found that a few terms in the infinite series are adequate for most practical purposes. Figures $2(\mathrm{a})-(\mathrm{c})$ show the variation of the dimensionless parallel surface displacement $(u / b)$ with the dimensionless horizontal distance from the upper edge of the fault $\left(x_{2} / H\right)$ for $\delta=15^{\circ}, 45^{\circ}, 75^{\circ}$, for four different values of $d=0, H / 10, H / 2$ and $3 H / 4$. We observe that the displacement for the surface-breaking fault $(d=0)$ is discontinuous at $x_{2}=0$. Figures $3(\mathrm{a})-(\mathrm{c})$ exhibit the variation of the dimensionless surface shear stress $P_{12}\left[=p_{12} /\left(\mu_{1} b / H\right)\right]$ with the distance from the fault $\left(x_{2} / H\right)$ for $\delta=15^{\circ}, 45^{\circ}, 75^{\circ}$. We observe that the variation of the shear stress for the surface breaking fault $(d=0)$ is remarkably different from the variation of the shear stress for buried faults.

\section{Acknowledgements}

Financial support from the University Grants Commission in the form of a Minor Research Project sanctioned to $\mathrm{S} R$ and from the Council of Scientific and Industrial Research and Indian National Science Academy to S J S is gratefully acknowledged. We are grateful for comments from the referee, which led to improvements in the presentation of the paper.

\section{References}

Chinnery M A and Jovanovich D B 1972 Effect of Earth layering on earthquake displacement fields; Bull. Seism. Soc. Am. 62 1629-1639.

Rybicki K 1971 The elastic residual field of a very long strike-slip fault in the presence of discontinuity; Bull. Seism. Soc. Am. 61 79-92. 
Savage J C and Prescott W H 1978 Asthenosphere readjustment and the earthquake cycle; J. Geophys. Res. 83 3369-3376.
Singh S J and Rani S 1994 Lithospheric deformation associated with two-dimensional strike-slip faulting; J. Phys. Earth. 42 197-220.

MS received 4 March 2004; accepted 23 August 2004 